\title{
Suzuki type theorems in triangular and non-Archimedean fuzzy metric spaces with application
}

\author{
Nawab Hussain ${ }^{1 *}$, Masoomeh Hezarjaribi ${ }^{2}$ and Peyman Salimi ${ }^{3}$
}

\section{"Correspondence:}

nhusain@kau.edu.sa

${ }^{1}$ Department of Mathematics, King

Abdulaziz University, P.O. Box 80203,

Jeddah, 21589, Saudi Arabia

Full list of author information is

available at the end of the article

\section{Springer}

\begin{abstract}
In this paper, first we introduce certain new classes of Suzuki type contractions in triangular and non-Archimedean fuzzy metric spaces. Further we establish fixed point theorems for such kind of mappings in non-Archimedean and triangular fuzzy metric spaces. We also prove Suzuki type fixed point results in non-Archimedean and triangular ordered fuzzy metric spaces. The results presented here improve and generalize certain recent results from the literature. Two illustrative examples and an application to integral equations are given to support the usability of our results.
\end{abstract}

MSC: $46 \mathrm{~N} 40 ; 47 \mathrm{H} 10 ; 54 \mathrm{H} 25 ; 46 \mathrm{~T} 99$

Keywords: fixed point; $\alpha$-admissible mapping; non-Archimedean fuzzy metric space; triangular fuzzy metric space

\section{Introduction and preliminaries}

The concept of fuzzy metric space was introduced in different ways by some authors (see, i.e., $[1,2])$ and further to this, the fixed point theory in this kind of spaces has been intensively studied (see [3-11]). Here, we consider the notion of fuzzy metric space introduced by Kramosil and Michálek [2] and modified by George and Veeramani [12,13] who obtained a Hausdorff topology for the class of fuzzy metric spaces. Recently, Miheț [14] enlarged the class of fuzzy contractive mappings of Gregori and Sapena [7] and proved a fuzzy Banach contraction result for complete non-Archimedean fuzzy metric spaces [7] (see also Vetro [15]).

The applications of fixed point theorems are remarkable in different disciplines of mathematics, engineering and economics in dealing with problems arising in approximation theory, game theory and many others (see [16] and the references therein). Consequently, many researchers, following the Banach contraction principle, investigated the existence of weaker contractive conditions or extended previous results under relatively weak hypotheses on the metric space. On the other hand, Samet et al. [17] introduced the concepts of $\alpha-\psi$-contractive and $\alpha$-admissible mappings and established various fixed point theorems for such mappings defined on complete metric spaces. Afterwards Salimi et al. [18] and Hussain et al. $[19,20]$ modified the notions of $\alpha$ - $\psi$-contractive and $\alpha$-admissible mappings and established certain fixed point theorems (see also [21-25]). In this paper,

(c) 2015 Hussain et al. This article is distributed under the terms of the Creative Commons Attribution 4.0 International License (http://creativecommons.org/licenses/by/4.0/), which permits unrestricted use, distribution, and reproduction in any medium, provided you give appropriate credit to the original author(s) and the source, provide a link to the Creative Commons license, and indicate if changes were made. 
we introduce certain new classes of contraction mappings and establish fixed point theorems for such kind of mappings in non-Archimedean fuzzy metric spaces. The results presented in this paper generalize and extend some recent results in non-Archimedean fuzzy metric spaces. Some examples are given to support the usability of our results. For the sake of completeness, we now briefly recall some basic concepts.

Definition 1.1 A binary operation $\star:[0,1] \times[0,1] \rightarrow[0,1]$ is called a continuous t-norm if it satisfies the following assertions:

$(\mathrm{TN} 1) \star$ is commutative and associative;

(TN2) $\star$ is continuous;

(TN3) $a \star 1=a$ for all $a \in[0,1]$;

(TN4) $a \star b \leq c \star d$ when $a \leq c$ and $b \leq d$ and $a, b, c, d \in[0,1]$.

Definition 1.2 (George and Veeramani [12]) A fuzzy metric space is an ordered triple $(X, M, \star)$ such that $X$ is a nonempty set, $\star$ is a continuous t-norm and $M$ is a fuzzy set on $X \times X \times(0,+\infty)$ satisfying the following conditions, for all $x, y, z \in X$ and $t, s>0$ :

(FM1) $M(x, y, t)>0$ for all $t>0$;

(FM2) $M(x, y, t)=1$ if and only if $x=y$;

(FM3) $M(x, y, t)=M(y, x, t)$;

(FM4) $M(x, y, t) \star M(y, z, s) \leq M(x, z, t+s)$;

(FM5) $M(x, y, \cdot):(0,+\infty) \rightarrow(0,1]$ is left continuous.

Then the triple $(X, M, \star)$ is called a fuzzy metric space. If we replace (FM4) by

(FM6) $M(x, y, t) \star M(y, z, s) \leq M(x, z, \max \{t, s\})$,

then the triple $(X, M, \star)$ is called a non-Archimedean fuzzy metric space. Since (FM6) implies (FM4), then each non-Archimedean fuzzy metric space is a fuzzy metric space.

Definition 1.3 Let $(X, M, \star)$ be a fuzzy metric space (or non-Archimedean fuzzy metric space). Then

(i) a sequence $\left\{x_{n}\right\}$ converges to $x \in X$ if and only if $\lim _{n \rightarrow+\infty} M\left(x_{n}, x, t\right)=1$ for all $t>0$;

(ii) a sequence $\left\{x_{n}\right\}$ in $X$ is a Cauchy sequence if and only if for all $\epsilon \in(0,1)$ and $t>0$, there exists $n_{0}$ such that $M\left(x_{n}, x_{m}, t\right)>1-\epsilon$ for all $m, n \geq n_{0}$;

(iii) the fuzzy metric space (or the non-Archimedean fuzzy metric space) is called complete if every Cauchy sequence converges to some $x \in X$.

If $(X, M, \star)$ is a fuzzy metric space and $(X, \preceq)$ is partially ordered, then $(X, M, \star)$ is called a partially ordered fuzzy metric space. Then $x, y \in X$ are called comparable if $x \preceq y$ or $y \preceq x$ holds. Let $f: X \rightarrow X$ be a mapping, $f$ is said to be non-decreasing if $f x \preceq f y$ whenever $x, y \in X$ and $x \leq y$.

Definition 1.4 [3] Let $(X, M, *)$ be a triangular fuzzy metric space. The fuzzy metric $M$ is called triangular whenever

$$
\frac{1}{M(x, y, t)}-1 \leq \frac{1}{M(x, z, t)}-1+\frac{1}{M(z, y, t)}-1 .
$$

Definition 1.5 [17] Let $f: X \rightarrow X$ and $\alpha: X \times X \rightarrow[0,+\infty) . f$ is an $\alpha$-admissible mapping if

$$
\alpha(x, y) \geq 1 \quad \text { implies } \quad \alpha(f x, f y) \geq 1, \quad x, y \in X .
$$


Definition 1.6 [26] Let $(X, M, *)$ be a fuzzy metric space, $T: X \rightarrow X$ and $\alpha: X \times X \times$ $(0, \infty) \rightarrow[0, \infty)$. We say that $T$ is an $\alpha$-admissible mapping if

$$
x, y \in X, \quad \alpha(x, y, t) \geq t \quad \Longrightarrow \quad \alpha(T x, T y, t) \geq t
$$

for all $t>0$.

\section{Fixed point results in triangular fuzzy metric spaces}

Let $(X, M, *)$ be a fuzzy metric space, $f: X \rightarrow X$ be a self-mapping on $X$. We define $P^{f}(x, y, t), Q^{f}(x, y, t)$ and $R^{f}(x, y, t)$ as follows:

$$
\begin{aligned}
& P^{f}(x, y, t)=\max \left\{\frac{1}{M(x, y, t)}, \frac{1}{M(x, f x, t)}, \frac{1}{M(y, f y, t)}, \frac{1}{2}\left[\frac{1}{M(x, f y, t)}+\frac{1}{M(y, f x, t)}-1\right]\right\}, \\
& Q^{f}(x, y, t)=\max \{M(x, f x, t), M(y, f y, t), M(x, f y, t), M(y, f x, t)\}
\end{aligned}
$$

and

$$
R^{f}(x, y, t)=\min \left\{1-\frac{1}{M(x, f x, t)}, 1-\frac{1}{M(y, f y, t)}, 1-\frac{1}{M(x, f y, t)}, 1-\frac{1}{M(y, f x, t)}\right\} .
$$

We now state and prove our first result of this section.

Theorem 2.1 Let $(X, M, *)$ be a complete triangular fuzzy metric space and $f$ be a selfmapping on $X$. Also suppose that $\alpha: X \times X \times[0, \infty) \rightarrow[0, \infty)$ is a mapping. Assume that the following assertions hold:

(i) there exists $x_{0} \in X$ such that $\alpha\left(x_{0}, f x_{0}, t\right) \geq t$ for all $t>0$;

(ii) $f$ is an $\alpha$-admissible mapping;

(iii) if $\left\{x_{n}\right\}$ is a sequence in $X$ such that $\alpha\left(x_{n}, x_{n+1}, t\right) \geq t$ for all $n \in \mathbb{N}$ and all $t>0$ with $x_{n} \rightarrow x$ as $n \rightarrow \infty$, then $\alpha\left(x_{n}, x, t\right) \geq t$ for all $n \in \mathbb{N} \cup\{0\}$;

(iv) for all $x, y \in X$ and all $t>0$ with $\frac{1}{1+\lambda}\left(\frac{1}{M(x, f x, t)}-1\right) \leq \frac{1}{M(x, y, t)}-1$, we have

$$
\frac{\alpha(x, y, t)}{t M(f x, f y, t)} \leq \lambda P^{f}(x, y, t)+\left|Q^{f}(x, y, t)-\lambda\right|+L R^{f}(x, y, t)
$$

where $\lambda \in(0,1)$ and $L \geq 0$.

Then $f$ has a fixed point.

Proof Let $x_{0} \in X$ be such that $\alpha\left(x_{0}, f x_{0}, t\right) \geq t$ for all $t>0$. Define a sequence $\left\{x_{n}\right\}$ in $X$ by $x_{n}=f^{n} x_{0}=f x_{n-1}$ for all $n \in \mathbb{N}$. If $x_{n+1}=x_{n}$ for some $n \in \mathbb{N}$, then $x=x_{n}$ is a fixed point for $f$ and the result is proved. Hence, we suppose that $x_{n+1} \neq x_{n}$ for all $n \in \mathbb{N}$. Since $f$ is an $\alpha$-admissible mapping and $\alpha\left(x_{0}, f x_{0}, t\right) \geq t$, we deduce that $\alpha\left(x_{1}, x_{2}, t\right)=\alpha\left(f x_{0}, f^{2} x_{0}, t\right) \geq t$. Continuing this process, we get

$$
\alpha\left(x_{n}, x_{n+1}, t\right) \geq t
$$

for all $n \in \mathbb{N} \cup\{0\}$ and all $t>0$. Now since

$$
\frac{1}{1+\lambda}\left(\frac{1}{M\left(x_{n-1}, f x_{n-1}, t\right)}-1\right) \leq \frac{1}{M\left(x_{n-1}, f x_{n-1}, t\right)}-1=\frac{1}{M\left(x_{n-1}, x_{n}, t\right)}-1,
$$


then from (iv) with $x=x_{n-1}$ and $y=x_{n}$ we get

$$
\begin{aligned}
\frac{1}{M\left(x_{n}, x_{n+1}, t\right)} & \leq \frac{\alpha\left(x_{n}, x_{n+1}, t\right)}{t M\left(x_{n}, x_{n+1}, t\right)}=\frac{\alpha\left(x_{n}, x_{n+1}, t\right)}{t M\left(f x_{n-1}, f x_{n}, t\right)} \\
& \leq \lambda P^{f}\left(x_{n-1}, x_{n}, t\right)+\left|Q^{f}\left(x_{n-1}, x_{n}, t\right)-\lambda\right|+L R^{f}\left(x_{n-1}, x_{n}, t\right) .
\end{aligned}
$$

That is,

$$
\frac{1}{M\left(x_{n}, x_{n+1}, t\right)} \leq \lambda P^{f}\left(x_{n-1}, x_{n}, t\right)+\left|Q^{f}\left(x_{n-1}, x_{n}, t\right)-\lambda\right|+L R^{f}\left(x_{n-1}, x_{n}, t\right)
$$

for all $t>0$ and all $n \in \mathbb{N}$, where

$$
\begin{aligned}
P^{f}\left(x_{n-1}, x_{n}, t\right)= & \max \left\{\frac{1}{M\left(x_{n-1}, x_{n}, t\right)}, \frac{1}{M\left(x_{n-1}, f x_{n-1}, t\right)},\right. \\
& \left.\frac{1}{M\left(x_{n}, f x_{n}, t\right)}, \frac{1}{2}\left[\frac{1}{M\left(x_{n-1}, f x_{n}, t\right)}+\frac{1}{M\left(x_{n}, f x_{n-1}, t\right)}-1\right]\right\} \\
= & \max \left\{\frac{1}{M\left(x_{n-1}, x_{n}, t\right)}, \frac{1}{M\left(x_{n}, x_{n+1}, t\right)}, \frac{1}{2 M\left(x_{n-1}, x_{n+1}, t\right)}\right\} \\
\leq & \max \left\{\frac{1}{M\left(x_{n-1}, x_{n}, t\right)}, \frac{1}{M\left(x_{n}, x_{n+1}, t\right)},\right. \\
& \left.\frac{1}{2}\left[\frac{1}{M\left(x_{n-1}, x_{n}, t\right)}-1+\frac{1}{M\left(x_{n}, x_{n+1}, t\right)}-1\right]+\frac{1}{2}\right\} \\
\leq & \max \left\{\frac{1}{M\left(x_{n-1}, x_{n}, t\right)}, \frac{1}{M\left(x_{n}, x_{n+1}, t\right)},\right. \\
& \left.\frac{1}{2}\left[\frac{1}{M\left(x_{n-1}, x_{n}, t\right)}+\frac{1}{M\left(x_{n}, x_{n+1}, t\right)}\right]-\frac{1}{2}\right\} \\
= & \max \left\{\frac{1}{M\left(x_{n-1}, x_{n}, t\right)}, \frac{1}{M\left(x_{n}, x_{n+1}, t\right)}\right\} \leq P^{f}\left(x_{n-1}, x_{n}, t\right),
\end{aligned}
$$

which implies

$$
P^{f}\left(x_{n-1}, x_{n}, t\right)=\max \left\{\frac{1}{M\left(x_{n-1}, x_{n}, t\right)}, \frac{1}{M\left(x_{n}, x_{n+1}, t\right)}\right\}
$$

and

$$
\begin{aligned}
Q^{f}\left(x_{n-1}, x_{n}, t\right) & =\max \left\{M\left(x_{n-1}, f x_{n-1}, t\right), M\left(x_{n}, f x_{n}, t\right), M\left(x_{n-1}, f x_{n}, t\right), M\left(x_{n}, f x_{n-1}, t\right)\right\} \\
& =\max \left\{M\left(x_{n-1}, x_{n}, t\right), M\left(x_{n}, x_{n+1}, t\right), M\left(x_{n-1}, x_{n+1}, t\right), M\left(x_{n}, x_{n}, t\right)\right\} \\
& =1 .
\end{aligned}
$$

Also,

$$
\begin{aligned}
R^{f}\left(x_{n-1}, x_{n}, t\right)= & \min \left\{1-\frac{1}{M\left(x_{n-1}, f x_{n-1}, t\right)}, 1-\frac{1}{M\left(x_{n}, f x_{n}, t\right)},\right. \\
& \left.1-\frac{1}{M\left(x_{n-1}, f x_{n}, t\right)}, 1-\frac{1}{M\left(x_{n}, f x_{n-1}, t\right)}\right\}
\end{aligned}
$$




$$
\begin{aligned}
= & \min \left\{1-\frac{1}{M\left(x_{n-1}, x_{n}, t\right)}, 1-\frac{1}{M\left(x_{n}, x_{n+1}, t\right)},\right. \\
& \left.1-\frac{1}{M\left(x_{n-1}, x_{n+1}, t\right)}, 1-\frac{1}{M\left(x_{n}, x_{n}, t\right)}\right\}=0
\end{aligned}
$$

From (2.3), (2.4), (2.5) and (2.6) we obtain

$$
\begin{aligned}
\frac{1}{M\left(x_{n}, x_{n+1}, t\right)} & \leq \lambda P^{f}\left(x_{n-1}, x_{n}, t\right)+|1-\lambda| \\
& =\lambda P^{f}\left(x_{n-1}, x_{n}, t\right)+1-\lambda \\
& =\lambda\left(P^{f}\left(x_{n-1}, x_{n}, t\right)-1\right)+1,
\end{aligned}
$$

which implies

$$
\frac{1}{M\left(x_{n}, x_{n+1}, t\right)}-1 \leq \lambda\left(P^{f}\left(x_{n-1}, x_{n}, t\right)-1\right) .
$$

Now if $P^{f}\left(x_{n-1}, x_{n}, t\right)=\frac{1}{M\left(x_{n}, x_{n+1}, t\right)}$, then we get

$$
\begin{aligned}
\frac{1}{M\left(x_{n}, x_{n+1}, t\right)}-1 & \leq \lambda\left[\frac{1}{M\left(x_{n}, x_{n+1}, t\right)}-1\right] \\
& <\frac{1}{M\left(x_{n}, x_{n+1}, t\right)}-1
\end{aligned}
$$

which is a contradiction. Hence,

$$
\frac{1}{M\left(x_{n}, x_{n+1}, t\right)}-1 \leq \lambda\left[\frac{1}{M\left(x_{n-1}, x_{n}, t\right)}-1\right]
$$

for all $n \in \mathbb{N}$ and all $t>0$. This implies

$$
\frac{1}{M\left(x_{n}, x_{n+1}, t\right)}-1 \leq \lambda^{n}\left[\frac{1}{M\left(x_{0}, x_{1}, t\right)}-1\right] .
$$

Thus, for all $n>m$, we have

$$
\begin{aligned}
\frac{1}{M\left(x_{n}, x_{m}, t\right)}-1 & \leq \frac{1}{M\left(x_{n}, x_{n-1}, t\right)}-1+\cdots+\frac{1}{M\left(x_{m+1}, x_{m}, t\right)}-1 \\
& \leq\left(\lambda^{n-1}+\lambda^{n-2}+\cdots+\lambda^{m}\right)\left(\frac{1}{M\left(x_{0}, x_{1}, t\right)}-1\right) \\
& \leq \frac{\lambda^{m}}{1-\lambda}\left(\frac{1}{M\left(x_{0}, x_{1}, t\right)}-1\right)
\end{aligned}
$$

That is, $\left\{x_{n}\right\}$ is a Cauchy sequence. Since, $X$ is a complete fuzzy metric space, then there exists $x^{*} \in X$ such that $x_{n} \rightarrow x^{*}$ as $n \rightarrow \infty$. By (iii), $\alpha\left(x_{n}, x^{*}, t\right) \geq t$ holds for all $n \in \mathbb{N}$ and all $t>0$. Suppose that there exists $n_{0} \in \mathbb{N}$ such that

$$
\frac{1}{1+\lambda}\left(\frac{1}{M\left(x_{n_{0}}, f x_{n_{0}}, t\right)}-1\right)>\frac{1}{M\left(x_{n_{0}}, x^{*}, t\right)}-1
$$


and

$$
\frac{1}{1+\lambda}\left(\frac{1}{M\left(x_{n_{0}-1}, f x_{n_{0}-1}, t\right)}-1\right)>\frac{1}{M\left(x_{n_{0}-1}, x^{*}, t\right)}-1
$$

By (2.7) we deduce

$$
\begin{aligned}
\frac{1}{M\left(x_{n_{0}-1}, f x_{n_{0}-1}, t\right)}-1= & \frac{1}{M\left(x_{n_{0}-1}, x_{n_{0}}, t\right)}-1 \\
\leq & \frac{1}{M\left(x_{n_{0}-1}, x, t\right)}-1+\frac{1}{M\left(x_{n_{0}}, x, t\right)}-1 \\
< & \frac{1}{1+\lambda}\left(\frac{1}{M\left(x_{n_{0}-1}, f x_{n_{0}-1}, t\right)}-1\right) \\
& +\frac{1}{1+\lambda}\left(\frac{1}{M\left(x_{n_{0}}, f x_{n_{0}}, t\right)}-1\right) \\
\leq & \frac{1}{1+\lambda}\left(\frac{1}{M\left(x_{n_{0}-1}, f x_{n_{0}-1}, t\right)}-1\right) \\
& +\frac{\lambda}{1+\lambda}\left(\frac{1}{M\left(x_{n_{0}-1}, f x_{n_{0}-1}, t\right)}-1\right) \\
= & \frac{1}{M\left(x_{n_{0}-1}, f x_{n_{0}-1}, t\right)}-1,
\end{aligned}
$$

which is a contradiction. Hence, either

$$
\frac{1}{1+r}\left(\frac{1}{M\left(x_{n}, f x_{n}, t\right)}-1\right) \leq \frac{1}{M\left(x_{n}, x^{*}, t\right)}-1
$$

or

$$
\frac{1}{1+r}\left(\frac{1}{M\left(x_{n-1}, f x_{n-1}, t\right)}-1\right) \leq \frac{1}{M\left(x_{n-1}, x^{*}, t\right)}-1
$$

holds for all $n \in \mathbb{N}$. Let

$$
\frac{1}{1+r}\left(\frac{1}{M\left(x_{n}, f x_{n}, t\right)}-1\right) \leq \frac{1}{M\left(x_{n}, x^{*}, t\right)}-1
$$

Then from (iv) we have

$$
\begin{aligned}
\frac{1}{M\left(x_{n}, f x^{*}, t\right)} & \leq \frac{\alpha\left(x_{n}, x^{*}, t\right)}{t M\left(x_{n+1}, f x^{*}, t\right)}=\frac{\alpha\left(x_{n}, x^{*}, t\right)}{t M\left(f x_{n}, f x^{*}, t\right)} \\
& \leq \lambda P^{f}\left(x_{n}, x^{*}, t\right)+\left|Q^{f}\left(x_{n}, x^{*}, t\right)-\lambda\right|
\end{aligned}
$$

for all $t>0$, where

$$
\begin{aligned}
P^{f}\left(x_{n}, x^{*}, t\right)= & \max \left\{\frac{1}{M\left(x_{n}, x^{*}, t\right)}, \frac{1}{M\left(x_{n}, f x_{n}, t\right)}, \frac{1}{M\left(x^{*}, f x^{*}, t\right)},\right. \\
& \left.\frac{1}{2}\left[\frac{1}{M\left(x_{n}, f x^{*}, t\right)}+\frac{1}{M\left(x^{*}, f x_{n}, t\right)}-1\right]\right\}
\end{aligned}
$$




$$
\begin{aligned}
= & \max \left\{\frac{1}{M\left(x_{n}, x^{*}, t\right)}, \frac{1}{M\left(x_{n}, x_{n+1}, t\right)}, \frac{1}{M\left(x^{*}, f x^{*}, t\right)},\right. \\
& \left.\frac{1}{2}\left[\frac{1}{M\left(x_{n}, f x^{*}, t\right)}+\frac{1}{M\left(x^{*}, x_{n+1}, t\right)}-1\right]\right\},
\end{aligned}
$$

and so

$$
\lim _{n \rightarrow \infty} P^{f}\left(x_{n}, x^{*}, t\right)=\frac{1}{M\left(x^{*}, f x^{*}, t\right)} .
$$

Also,

$$
Q^{f}\left(x_{n}, x^{*}, t\right)=\max \left\{M\left(x_{n}, x_{n+1}, t\right), M\left(x^{*}, f x^{*}, t\right), M\left(x_{n}, f x^{*}, t\right), M\left(x^{*}, x_{n+1}, t\right)\right\},
$$

and so

$$
\lim _{n \rightarrow \infty} Q^{f}\left(x_{n}, x^{*}, t\right)=1
$$

Similarly,

$$
\lim _{n \rightarrow \infty} R^{f}\left(x_{n}, x^{*}, t\right)=0
$$

By taking limit as $n \rightarrow \infty$ in (2.10) we get

$$
\frac{1}{M\left(x^{*}, f x^{*}, t\right)}-1 \leq \lambda\left(\frac{1}{M\left(x^{*}, f x^{*}, t\right)}-1\right)
$$

for all $t>0$. Now, assume that there exists $t_{0}$ such that $M\left(x^{*}, f x^{*}, t_{0}\right)<1$. Then by the above inequality we have $1 \leq \lambda$, which is a contradiction. Hence, $M\left(x^{*}, f x^{*}, t\right)=1$ for all $t>0$; i.e., $x^{*}=f x^{*}$. Similarly we can deduce that $x^{*}$ is a fixed point of $f$ when

$$
\frac{1}{1+r}\left(\frac{1}{M\left(x_{n-1}, f x_{n-1}, t\right)}-1\right) \leq \frac{1}{M\left(x_{n-1}, x^{*}, t\right)}-1 .
$$

Example 2.1 Let $X=\mathbb{R}^{2}$. We define $\alpha: X \times X \times[0, \infty) \rightarrow[0, \infty)$ by

$$
\alpha(x, y, t)= \begin{cases}t, & x, y \in U=\{(0,0),(4,0),(0,4),(4,5),(5,4)\}, \\ 0, & \text { otherwise }\end{cases}
$$

Define $M$ on $X \times X \times(0, \infty)$ by $M\left(\left(x_{1}, x_{2}\right),\left(y_{1}, y_{2}\right), t\right)=\frac{1}{1+\left|x_{1}-y_{1}\right|+\left|x_{2}-y_{2}\right|}$ and $a \star b=\min \{a, b\}$. Clearly, $(M, X, \star)$ is a complete triangular fuzzy metric space. Also, define $f: X \rightarrow X$ and $\psi:[0, \infty) \rightarrow[0, \infty)$ by

$$
f\left(x_{1}, x_{2}\right)=\left\{\begin{array}{ll}
\left(x_{1}, 0\right) & \text { if } x_{1} \leq x_{2} \text { and } x_{1}, x_{2} \in U, \\
\left(0, x_{2}\right) & \text { if } x_{1}>x_{2} \text { and } x_{1}, x_{2} \in U, \\
\left(2 x_{1}^{2}, 3 x_{2}^{3}\right) & \text { if } x_{1}, x_{2} \in \mathbb{R}^{2} \backslash U
\end{array} \quad \text { and } \quad \psi(t)=0.99 t\right.
$$

First we assume

$$
\frac{1}{1+\frac{9}{10}}\left(\frac{1}{M(x, f x, t)}-1\right)=\frac{10}{19}\left(\frac{1}{M(x, f x, t)}-1\right) \leq \frac{1}{M(x, y, t)}-1
$$


and $\alpha(x, y, t) \geq t($ or $x, y \in U)$. Then

$$
\begin{aligned}
(x, y) \in\{ & ((0,0),(4,0)),((0,0),(0,4)),((0,0),(4,5)),((0,0),(5,4)), \\
& ((4,0),(0,0)),((4,0),(0,4)),((4,0),(5,4)),((4,0),(4,5)), \\
& ((0,4),(0,0)),((0,4),(4,0)),((0,4),(5,4)),((0,4),(4,5)), \\
& ((4,5),(0,0)),((4,5),(4,0)),((4,5),(0,4)),((4,5),(5,4)) \\
& ((5,4),(0,0)),((5,4),(4,0)),((5,4),(0,4)),((5,4),(4,5))\} .
\end{aligned}
$$

Since $M(f x, f y, t)=M(f y, f x, t), P^{f}(x, y, t)=P^{f}(y, x, t)$ and $Q^{f}(x, y, t)=Q^{f}(y, x, t)$, hence without any loss of generality we can reduce the above set to the following:

$$
\begin{aligned}
&(x, y) \in\{((0,0),(4,0)),((0,0),(0,4)),((0,0),(4,5)),((0,0),(5,4)), \\
&((4,0),(0,4)),((4,0),(5,4)),((4,0),(4,5)),((0,4),(5,4)),((0,4),(4,5))\} .
\end{aligned}
$$

Now, we consider the following cases:

- Let $(x, y)=((0,0),(4,0))$, then

$$
\begin{aligned}
P^{f}((0,0),(4,0), t)= & \max \left\{\frac{1}{M((0,0),(4,0), t)}, \frac{1}{M((0,0), f(0,0), t)}, \frac{1}{M((4,0), f(4,0), t)},\right. \\
& \left.\frac{1}{2}\left[\frac{1}{M((0,0), f(4,0), t)}+\frac{1}{M((4,0), f(0,0), t)}-1\right]\right\} \\
= & \max \left\{\frac{1}{M((0,0),(4,0), t)}, \frac{1}{M((0,0),(0,0), t)}, \frac{1}{M((4,0),(0,0), t)},\right. \\
& \left.\frac{1}{2}\left[\frac{1}{M((0,0),(0,0), t)}+\frac{1}{M((4,0),(0,0), t)}-1\right]\right\} \\
= & \max \left\{5,1,5, \frac{1}{2}[1+5-1]\right\}=5
\end{aligned}
$$

and

$$
\begin{aligned}
Q^{f}((0,0),(4,0), t)= & \max \{M((0,0), f(0,0), t), M((4,0), f(4,0), t), \\
& M((0,0), f(4,0), t), M((4,0), f(0,0), t)\} \\
= & \max \{M((0,0),(0,0), t), M((4,0),(0,0), t), \\
& M((0,0),(0,0), t), M((4,0),(0,0), t)\} \\
= & \max \left\{1, \frac{1}{5}, 1, \frac{1}{5}\right\}=1,
\end{aligned}
$$

and so

$$
\begin{aligned}
\frac{\alpha((0,0),(4,0), t)}{t M(f(0,0), f(4,0), t)} & =1 \leq \frac{46}{10}=\frac{9}{10} \cdot 5+\left|1-\frac{9}{10}\right| \\
& =\frac{9}{10} P^{f}((0,0),(4,0), t)+\left|Q^{f}((0,0),(4,0), t)-\frac{9}{10}\right|
\end{aligned}
$$


- Let $(x, y)=((0,0),(0,4))$, then

$$
\begin{aligned}
P^{f}((0,0),(4,0), t)= & \max \left\{\frac{1}{M((0,0),(4,0), t)}, \frac{1}{M((0,0), f(0,0), t)}, \frac{1}{M((4,0), f(4,0), t)},\right. \\
& \left.\frac{1}{2}\left[\frac{1}{M((0,0), f(4,0), t)}+\frac{1}{M((4,0), f(0,0), t)}-1\right]\right\} \\
= & \max \left\{\frac{1}{M((0,0),(4,0), t)}, \frac{1}{M((0,0),(0,0), t)}, \frac{1}{M((4,0),(0,0), t)},\right. \\
& \left.\frac{1}{2}\left[\frac{1}{M((0,0),(0,0), t)}+\frac{1}{M((4,0),(0,0), t)}-1\right]\right\} \\
= & \max \left\{5,1,5, \frac{1}{2}[1+5-1]\right\}=5
\end{aligned}
$$

and

$$
\begin{aligned}
Q^{f}((0,0),(4,0), t)= & \max \{M((0,0), f(0,0), t), M((4,0), f(4,0), t), \\
& M((0,0), f(4,0), t), M((4,0), f(0,0), t)\} \\
= & \max \{M((0,0),(0,0), t), M((4,0),(0,0), t), \\
& M((0,0),(0,0), t), M((4,0),(0,0), t)\} \\
= & \max \left\{1, \frac{1}{5}, 1, \frac{1}{5}\right\}=1,
\end{aligned}
$$

and so

$$
\begin{aligned}
\frac{\alpha((0,0),(4,0), t)}{t M(f(0,0), f(4,0), t)} & =1 \leq \frac{46}{10}=\frac{9}{10} \cdot 5+\left|1-\frac{9}{10}\right| \\
& =\frac{9}{10} P^{f}((0,0),(4,0), t)+\left|Q^{f}((0,0),(4,0), t)-\frac{9}{10}\right| .
\end{aligned}
$$

- Let $(x, y)=((0,0),(4,5))$, then

$$
\begin{aligned}
P^{f}((0,0),(4,5), t)= & \max \left\{\frac{1}{M((0,0),(4,5), t)}, \frac{1}{M((0,0), f(0,0), t)}, \frac{1}{M((4,5), f(4,5), t)},\right. \\
& \left.\frac{1}{2}\left[\frac{1}{M((0,0), f(4,5), t)}+\frac{1}{M((4,5), f(0,0), t)}-1\right]\right\} \\
= & \max \left\{\frac{1}{M((0,0),(4,5), t)}, \frac{1}{M((0,0),(0,0), t)}, \frac{1}{M((4,5),(4,0), t)},\right. \\
& \left.\frac{1}{2}\left[\frac{1}{M((0,0),(4,0), t)}+\frac{1}{M((4,5),(0,0), t)}-1\right]\right\} \\
= & \max \left\{10,1,6, \frac{1}{2}[5+10-1]\right\}=10
\end{aligned}
$$

and

$$
\begin{aligned}
Q^{f}((0,0),(4,5), t)= & \max \{M((0,0), f(0,0), t), M((4,5), f(4,5), t) \\
& M((0,0), f(4,5), t), M((4,5), f(0,0), t)\}
\end{aligned}
$$




$$
\begin{aligned}
= & \max \{M((0,0),(0,0), t), M((4,5),(4,0), t), \\
& M((0,0),(4,0), t), M((4,5),(0,0), t)\} \\
= & \max \left\{1, \frac{1}{6}, \frac{1}{5}, \frac{1}{10}\right\}=1,
\end{aligned}
$$

and so

$$
\begin{aligned}
\frac{\alpha((0,0),(4,5), t)}{t M(f(0,0), f(4,5), t)} & =5 \leq \frac{91}{10}=\frac{9}{10} \cdot 10+\left|1-\frac{9}{10}\right| \\
& =\frac{9}{10} P^{f}((0,0),(4,5), t)+\left|Q^{f}((0,0),(4,5), t)-\frac{9}{10}\right|
\end{aligned}
$$

- Let $(x, y)=((0,0),(5,4))$, then

$$
\begin{aligned}
P^{f}((0,0),(5,4), t)= & \max \left\{\frac{1}{M((0,0),(5,4), t)}, \frac{1}{M((0,0), f(0,0), t)}, \frac{1}{M((5,4), f(5,4), t)}\right. \\
& \left.\frac{1}{2}\left[\frac{1}{M((0,0), f(5,4), t)}+\frac{1}{M((5,4), f(0,0), t)}-1\right]\right\} \\
= & \max \left\{\frac{1}{M((0,0),(5,4), t)}, \frac{1}{M((0,0),(0,0), t)}, \frac{1}{M((5,4),(0,4), t)}\right. \\
& \left.\frac{1}{2}\left[\frac{1}{M((0,0),(0,4), t)}+\frac{1}{M((5,4),(0,0), t)}-1\right]\right\} \\
= & \max \left\{10,1,6, \frac{1}{2}[5+10-1]\right\}=10
\end{aligned}
$$

and

$$
\begin{aligned}
Q^{f}((0,0),(5,4), t)= & \max \{M((0,0), f(0,0), t), M((5,4), f(5,4), t), \\
& M((0,0), f(5,4), t), M((5,4), f(0,0), t)\} \\
= & \max \{M((0,0),(0,0), t), M((5,4),(0,4), t), \\
& M((0,0),(0,4), t), M((5,4),(0,0), t)\} \\
= & \max \left\{1, \frac{1}{6}, \frac{1}{5}, \frac{1}{10}\right\}=1,
\end{aligned}
$$

and so

$$
\begin{aligned}
\frac{\alpha((0,0),(5,4), t)}{t M(f(0,0), f(5,4), t)} & =5 \leq \frac{91}{10}=\frac{9}{10} \cdot 10+\left|1-\frac{9}{10}\right| \\
& =\frac{9}{10} P^{f}((0,0),(5,4), t)+\left|Q^{f}((0,0),(5,4), t)-\frac{9}{10}\right|
\end{aligned}
$$

- Let $(x, y)=((4,0),(0,4))$, then

$$
\begin{aligned}
P^{f}((4,0),(0,4), t)= & \max \left\{\frac{1}{M((4,0),(0,4), t)}, \frac{1}{M((4,0), f(4,0), t)}, \frac{1}{M((0,4), f(0,4), t)},\right. \\
& \left.\frac{1}{2}\left[\frac{1}{M((4,0), f(0,4), t)}+\frac{1}{M((0,4), f(4,0), t)}-1\right]\right\}
\end{aligned}
$$




$$
\begin{aligned}
= & \max \left\{\frac{1}{M((4,0),(0,4), t)}, \frac{1}{M((4,0),(0,0), t)}, \frac{1}{M((0,4),(0,0), t)},\right. \\
& \left.\frac{1}{2}\left[\frac{1}{M((4,0),(0,0), t)}+\frac{1}{M((0,4),(0,0), t)}-1\right]\right\} \\
= & \max \left\{9,5,5, \frac{1}{2}[5+5-1]\right\}=9
\end{aligned}
$$

and

$$
\begin{aligned}
Q^{f}((4,0),(0,4), t)= & \max \{M((4,0), f(4,0), t), M((0,4), f(0,4), t), \\
& M((4,0), f(0,4), t), M((0,4), f(4,0), t)\} \\
= & \max \{M((4,0),(0,0), t), M((0,4),(0,0), t), \\
& M((4,0),(0,0), t), M((0,4),(0,0), t)\} \\
= & \max \left\{\frac{1}{5}, \frac{1}{5}, \frac{1}{5}, \frac{1}{5}\right\}=\frac{1}{5},
\end{aligned}
$$

and so

$$
\begin{aligned}
\frac{\alpha((4,0),(0,4), t)}{t M(f(4,0), f(0,4), t)} & =1 \leq \frac{88}{10}=\frac{9}{10} \cdot 9+\left|\frac{1}{5}-\frac{9}{10}\right| \\
& =\frac{9}{10} P^{f}((4,0),(0,4), t)+\left|Q^{T}((4,0),(0,4), t)-\frac{9}{10}\right| .
\end{aligned}
$$

- Let $(x, y)=((4,0),(5,4))$, then

$$
\begin{aligned}
P_{f}((4,0),(5,4), t)= & \max \left\{\frac{1}{M((4,0),(5,4), t)}, \frac{1}{M((4,0), f(4,0), t)}, \frac{1}{M((5,4), f(5,4), t)},\right. \\
& \left.\frac{1}{2}\left[\frac{1}{M((4,0), f(5,4), t)}+\frac{1}{M((5,4), f(4,0), t)}-1\right]\right\} \\
= & \max \left\{\frac{1}{M((4,0),(5,4), t)}, \frac{1}{M((4,0),(0,0), t)}, \frac{1}{M((5,4),(0,4), t)},\right. \\
& \left.\frac{1}{2}\left[\frac{1}{M((4,0),(0,4), t)}+\frac{1}{M((5,4),(0,0), t)}-1\right]\right\} \\
= & \max \left\{6,5,6, \frac{1}{2}[9+10-1]\right\}=9
\end{aligned}
$$

and

$$
\begin{aligned}
Q^{f}((4,0),(5,4), t)= & \max \{M((4,0), f(4,0), t), M((5,4), f(5,4), t), \\
& M((4,0), f(5,4), t), M((5,4), f(4,0), t)\} \\
= & \max \{M((4,0),(0,0), t), M((5,4),(0,4), t), \\
& M((4,0),(0,4), t), M((5,4),(0,0), t)\} \\
= & \max \left\{\frac{1}{5}, \frac{1}{6}, \frac{1}{9}, \frac{1}{10}\right\}=\frac{1}{5},
\end{aligned}
$$


and so

$$
\begin{aligned}
\frac{\alpha((4,0),(5,4), t)}{t M(f(4,0), f(5,4), t)} & =5 \leq \frac{88}{10}=\frac{9}{10} \cdot 9+\left|\frac{1}{5}-\frac{9}{10}\right| \\
& =\frac{9}{10} P^{f}((4,0),(5,4), t)+\left|Q^{f}((4,0),(5,4), t)-\frac{9}{10}\right| .
\end{aligned}
$$

- Let $(x, y)=((4,0),(4,5))$, then

$$
\begin{aligned}
P^{f}((4,0),(4,5), t)= & \max \left\{\frac{1}{M((4,0),(4,5), t)}, \frac{1}{M((4,0), f(4,0), t)}, \frac{1}{M((4,5), f(4,5), t)},\right. \\
& \left.\frac{1}{2}\left[\frac{1}{M((4,0), f(4,5), t)}+\frac{1}{M((4,5), f(4,0), t)}-1\right]\right\} \\
= & \max \left\{\frac{1}{M((4,0),(4,5), t)}, \frac{1}{M((4,0),(0,0), t)}, \frac{1}{M((4,5),(4,0), t)},\right. \\
& \left.\frac{1}{2}\left[\frac{1}{M((4,0),(4,0), t)}+\frac{1}{M((4,5), f(4,0), t)}-1\right]\right\} \\
= & \max \left\{6,5,6, \frac{1}{2}[1+6-1]\right\}=6
\end{aligned}
$$

and

$$
\begin{aligned}
Q^{f}((4,0),(4,5), t)= & \max \{M((4,0), f(4,0), t), M((4,5), f(4,5), t), \\
& M((4,0), f(4,5), t), M((4,5), f(4,0), t)\} \\
= & \max \{M((4,0),(0,0), t), M((4,5),(4,0), t), \\
& M((4,0),(4,0), t), M((4,5),(0,0), t)\} \\
= & \max \left\{\frac{1}{5}, \frac{1}{6}, 1, \frac{1}{10}\right\}=1,
\end{aligned}
$$

and so

$$
\begin{aligned}
\frac{\alpha((4,0),(5,4), t)}{t M(f(4,0), f(5,4), t)} & =5 \leq \frac{88}{10}=\frac{9}{10} \cdot 9+\left|\frac{1}{5}-\frac{9}{10}\right| \\
& =\frac{9}{10} P^{f}((4,0),(5,4), t)+\left|Q^{f}((4,0),(5,4), t)-\frac{9}{10}\right|
\end{aligned}
$$

- Let $(x, y)=((0,4),(5,4))$, then

$$
\begin{aligned}
P^{f}((0,4),(5,4), t)= & \max \left\{\frac{1}{M((0,4),(5,4), t)}, \frac{1}{M((0,4), f(0,4), t)}, \frac{1}{M((5,4), f(5,4), t)},\right. \\
& \left.\frac{1}{2}\left[\frac{1}{M((0,4), f(5,4), t)}+\frac{1}{M((5,4), f(0,4), t)}-1\right]\right\} \\
= & \max \left\{\frac{1}{M((0,4),(5,4), t)}, \frac{1}{M((0,4),(0,0), t)}, \frac{1}{M((5,4),(0,4), t)},\right. \\
& \left.\frac{1}{2}\left[\frac{1}{M((0,4),(0,4), t)}+\frac{1}{M((5,4),(0,0), t)}-1\right]\right\} \\
= & \max \left\{6,5,6, \frac{1}{2}[1+10-1]\right\}=6
\end{aligned}
$$


and

$$
\begin{aligned}
Q^{f}((0,4),(5,4), t)= & \max \{M((0,4), f(0,4), t), M((5,4), f(5,4), t), \\
& M((0,4), f(5,4), t), M((5,4), f(0,4), t)\} \\
= & \max \{M((0,4),(0,0), t), M((5,4),(0,4), t), \\
& M((0,4),(0,4), t), M((5,4),(0,0), t)\} \\
= & \max \left\{\frac{1}{5}, \frac{1}{6}, 1, \frac{1}{10}\right\}=1,
\end{aligned}
$$

and so

$$
\begin{aligned}
\frac{\alpha((0,4),(5,4), t)}{t M(f(0,4), f(5,4), t)} & =5 \leq \frac{55}{10}=\frac{9}{10} \cdot 6+\left|1-\frac{9}{10}\right| \\
& =\frac{9}{10} P^{f}((0,4),(5,4), t)+\left|Q^{f}((0,4),(5,4), t)-\frac{9}{10}\right|
\end{aligned}
$$

- Let $(x, y)=((0,4),(4,5))$, then

$$
\begin{aligned}
P^{f}((0,4),(4,5), t)= & \max \left\{\frac{1}{M((0,4),(4,5), t)}, \frac{1}{M((0,4), f(0,4), t)}, \frac{1}{M((4,5), f(4,5), t)},\right. \\
& \left.\frac{1}{2}\left[\frac{1}{M((0,4), f(4,5), t)}+\frac{1}{M((4,5), f(0,4), t)}-1\right]\right\} \\
= & \max \left\{\frac{1}{M((0,4),(4,5), t)}, \frac{1}{M((0,4),(0,0), t)}, \frac{1}{M((4,5),(4,0), t)},\right. \\
& \left.\frac{1}{2}\left[\frac{1}{M((0,4),(4,0), t)}+\frac{1}{M((4,5),(0,0), t)}-1\right]\right\} \\
= & \max \left\{6,5,6, \frac{1}{2}[9+10-1]\right\}=9
\end{aligned}
$$

and

$$
\begin{aligned}
Q^{f}((0,4),(4,5), t)= & \max \{M((0,4), f(0,4), t), M((4,5), f(4,5), t), \\
& M((0,4), f(4,5), t), M((4,5), f(0,4), t)\} \\
= & \max \{M((0,4),(0,0), t), M((4,5),(4,0), t), \\
& M((0,4),(4,0), t), M((4,5),(0,0), t)\} \\
= & \max \left\{\frac{1}{5}, \frac{1}{6}, \frac{1}{9}, \frac{1}{10}\right\}=\frac{1}{5},
\end{aligned}
$$

and so

$$
\begin{aligned}
\frac{\alpha((0,4),(4,5), t)}{t M(f(0,4), f(4,5), t)} & =5 \leq \frac{88}{10}=\frac{9}{10} \cdot 9+\left|\frac{1}{5}-\frac{9}{10}\right| \\
& =\frac{9}{10} P^{f}((0,4),(4,5), t)+\left|Q^{f}((0,4),(4,5), t)-\frac{9}{10}\right|
\end{aligned}
$$


Otherwise, $\alpha(x, y, t)=0$, and so

$$
\frac{\alpha(x, y, t)}{t M(f x, f y, t)}=0 \leq \frac{9}{10} P^{f}(x, y, t)+\left|Q^{f}(x, y, t)-\frac{9}{10}\right|
$$

That is,

$$
\frac{1}{1+\frac{9}{10}}\left(\frac{1}{M(x, f x, t)}-1\right) \leq \frac{1}{M(x, y, t)}-1
$$

implies

$$
\begin{aligned}
\frac{\alpha(x, y, t)}{t M(f x, f y, t)} & \leq \frac{9}{10} P^{f}(x, y, t)+\left|Q^{f}(x, y, t)-\frac{9}{10}\right| \\
& \leq \frac{9}{10} P^{f}(x, y, t)+\left|Q^{f}(x, y, t)-\frac{9}{10}\right|+L R^{f}(x, y, t),
\end{aligned}
$$

where $L \geq 0$. Let $\alpha(x, y, t) \geq t$, then $x, y \in U$. On the other hand, $f w \in U$ for all $w \in U$. Then $\alpha(f x, f y, t) \geq t$. That is, $f$ is an $\alpha$-admissible mapping. If $\left\{x_{n}\right\}$ is a sequence in $X$ such that $\alpha\left(x_{n}, x_{n+1}, t\right) \geq t$ with $x_{n} \rightarrow x$ as $n \rightarrow \infty$, then $x_{n} \in U$ for all $n \in \mathbb{N}$. Also, $U$ is a closed set, so $x \in U$. That is, $\alpha\left(x_{n}, x, t\right) \geq t$ for all $n \in \mathbb{N} \cup\{0\}$. Clearly, $\alpha((0,0), f(0,0), t) \geq t$.

Therefore all conditions of Theorem 2.1 hold and $f$ has a fixed point. Here, $x=(0,0)$ is a fixed point of $f$.

Corollary 2.1 Let $(X, M, *)$ be a complete triangular fuzzy metric space and $f$ be a selfmapping on $X$. Also suppose that $\alpha: X \times X \times[0, \infty) \rightarrow[0, \infty)$ is a mapping. Assume that the following assertions hold:

(i) there exists $x_{0} \in X$ such that $\alpha\left(x_{0}, f x_{0}, t\right) \geq t$ for all $t>0$;

(ii) $f$ is an $\alpha$-admissible mapping;

(iii) if $\left\{x_{n}\right\}$ is a sequence in $X$ such that $\alpha\left(x_{n}, x_{n+1}, t\right) \geq t$ for all $n \in \mathbb{N}$ and all $t>0$ with $x_{n} \rightarrow x$ as $n \rightarrow \infty$, then $\alpha\left(x, x_{n}, t\right) \geq t$ for all $n \in \mathbb{N} \cup\{0\}$

(iv) for all $x, y \in X$ and all $t>0$, we have

$$
\frac{\alpha(x, y, t)}{t M(f x, f y, t)} \leq \lambda P^{f}(x, y, t)+\left|Q^{f}(x, y, t)-\lambda\right|+L R^{f}(x, y, t)
$$

holds where $\lambda \in(0,1)$ and $L \geq 0$.

Then $f$ has a fixed point.

By taking $L=0$ in Corollary 2.1, we obtain the following corollary.

Corollary 2.2 Let $(X, M, *)$ be a complete triangular fuzzy metric space and $f$ be a selfmapping on $X$. Also suppose that $\alpha: X \times X \times[0, \infty) \rightarrow[0, \infty)$ is a mapping. Assume that the following assertions hold:

(i) there exists $x_{0} \in X$ such that $\alpha\left(x_{0}, f x_{0}, t\right) \geq t$ for all $t>0$;

(ii) $f$ is an $\alpha$-admissible mapping;

(iii) if $\left\{x_{n}\right\}$ is a sequence in $X$ such that $\alpha\left(x_{n}, x_{n+1}, t\right) \geq t$ for all $n \in \mathbb{N}$ and all $t>0$ with $x_{n} \rightarrow x$ as $n \rightarrow \infty$, then $\alpha\left(x, x_{n}, t\right) \geq$ f for all $n \in \mathbb{N} \cup\{0\}$; 
(iv) for all $x, y \in X$ and all $t>0$, we have

$$
\frac{\alpha(x, y, t)}{t M(f x, f y, t)} \leq \lambda P^{f}(x, y, t)+\left|Q^{f}(x, y, t)-\lambda\right|
$$

holds where $\lambda \in(0,1)$.

Then $f$ has a fixed point.

By taking $\alpha(x, y, t)=t$ for all $x, y \in X$ and all $t>0$ in Corollary 2.1, we obtain the following result.

Corollary 2.3 Let $(X, M, *)$ be a complete triangular fuzzy metric space and $f$ be a selfmapping on $X$. Assume that for all $x, y \in X$ and all $t>0$,

$$
\frac{1}{M(T x, T y, t)} \leq \lambda P^{f}(x, y, t)+\left|Q^{f}(x, y, t)-\lambda\right|+L R^{f}(x, y, t)
$$

holds where $\lambda \in(0,1)$ and $L \geq 0$. Then $f$ has a fixed point.

By taking $L=0$ in Corollary 2.3, we obtain the following corollary.

Corollary 2.4 Let $(X, M, *)$ be a complete triangular fuzzy metric space and $f$ be a selfmapping on $X$. Assume that for all $x, y \in X$ and all $t>0$,

$$
\frac{1}{M(f x, f y, t)} \leq \lambda P^{f}(x, y, t)+\left|Q^{f}(x, y, t)-\lambda\right|
$$

holds where $\lambda \in(0,1)$. Then $f$ has a fixed point.

Theorem 2.2 Let $(X, M, *, \preceq)$ be a complete triangular partially ordered fuzzy metric space and $f$ be a self-mapping on $X$. Assume that the following assertions hold:

(i) there exists $x_{0} \in X$ such that $x_{0} \preceq f x_{0}$;

(ii) $f$ is an increasing mapping;

(iii) if $\left\{x_{n}\right\}$ is an increasing sequence in $X$ such that with $x_{n} \rightarrow x$ as $n \rightarrow \infty$, then $x \leq x_{n}$ for all $n \in \mathbb{N} \cup\{0\}$;

(iv) for all $x, y \in X$ and all $t>0$ with $\frac{1}{1+\lambda}\left(\frac{1}{M(x, f x, t)}-1\right) \leq \frac{1}{M(x, y, t)}-1$ and $x \leq y$, we have

$$
\frac{1}{M(f x, f y, t)} \leq \lambda P^{f}(x, y, t)+\left|Q^{f}(x, y, t)-\lambda(t)\right|+L R^{f}(x, y, t)
$$

where $\lambda \in(0,1)$ and $L \geq 0$.

Then $f$ has a fixed point.

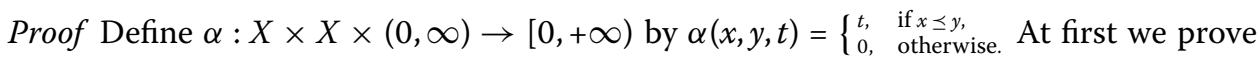
that $f$ is an $\alpha$-admissible mapping. Let $\alpha(x, y, t) \geq t$, then $x \leq y$. Now, since $f$ is increasing, then we have $f x \preceq f y$. That is, $\alpha(f x, f y, t) \geq t$. Therefore $f$ is an $\alpha$-admissible mapping. From (i) there exists $x_{0} \in X$ such that $x_{0} \preceq f x_{0}$. That is, $\alpha\left(x_{0}, f x_{0}, t\right) \geq t$ for all $t>0$. If $\left\{x_{n}\right\}$ is a sequence in $X$ such that $\alpha\left(x_{n}, x_{n+1}, t\right) \geq t$ for all $n \in \mathbb{N}$ and all $t>0$ with $x_{n} \rightarrow x$ as $n \rightarrow \infty$, then $x_{n} \preceq x_{n+1}$ for all $n \in \mathbb{N}$ with $x_{n} \rightarrow x$ as $n \rightarrow \infty$. Hence from (iii) we get $x_{n} \preceq x$ for all $n \in \mathbb{N} \cup\{0\}$. That is, $\alpha\left(x_{n}, x, t\right) \geq t$ for all $n \in \mathbb{N} \cup\{0\}$. 
Let $\frac{1}{1+\lambda}\left(\frac{1}{M(x, f x, t)}-1\right) \leq \frac{1}{M(x, y, t)}-1$ and $x \preceq y($ or $\alpha(x, y, t)=t)$, then from (iv) we have

$$
\frac{1}{M(f x, f y, t)} \leq \lambda P^{f}(x, y, t)+\left|Q^{f}(x, y, t)-\lambda(t)\right|+L R^{f}(x, y, t) .
$$

Also if $\alpha(x, y, t)=0$, then

$$
\frac{\alpha(x, y, t)}{t M(f x, f y, t)}=0 \leq \lambda P^{f}(x, y, t)+\left|Q^{f}(x, y, t)-\lambda(t)\right|+L R^{f}(x, y, t)
$$

That is, for all $x, y \in X$ and all $t>0$ with $\frac{1}{1+\lambda}\left(\frac{1}{M(x, f x, t)}-1\right) \leq \frac{1}{M(x, y, t)}-1$, we have

$$
\frac{\alpha(x, y, t)}{t M(f x, f y, t)} \leq \lambda P^{f}(x, y, t)+\left|Q^{f}(x, y, t)-\lambda(t)\right|+L R^{f}(x, y, t) .
$$

Hence, all conditions of Theorem 2.1 are satisfied and $f$ has a fixed point.

Corollary 2.5 Let $(X, M, *, \preceq)$ be a complete triangular partially ordered fuzzy metric space and $f$ be a self-mapping on $X$. Assume that the following assertions hold:

(i) there exists $x_{0} \in X$ such that $x_{0} \preceq f x_{0}$;

(ii) $f$ is an increasing mapping;

(iii) if $\left\{x_{n}\right\}$ is an increasing sequence in $X$ such that with $x_{n} \rightarrow x$ as $n \rightarrow \infty$, then $x \preceq x_{n}$ for all $n \in \mathbb{N} \cup\{0\}$;

(iv) for all $x, y \in X$ and all $t>0$ with $x \preceq y$, we have

$$
\frac{1}{M(f x, f y, t)} \leq \lambda P^{f}(x, y, t)+\left|Q^{f}(x, y, t)-\lambda\right|+L R^{f}(x, y, t)
$$

where $\lambda \in(0,1)$ and $L \geq 0$.

Then $f$ has a fixed point.

Corollary 2.6 Let $(X, M, *, \preceq)$ be a complete triangular partially ordered fuzzy metric space and $f$ be a self-mapping on $X$. Assume that the following assertions hold:

(i) there exists $x_{0} \in X$ such that $x_{0} \preceq f x_{0}$;

(ii) $f$ is an increasing mapping;

(iii) if $\left\{x_{n}\right\}$ is an increasing sequence in $X$ such that with $x_{n} \rightarrow x$ as $n \rightarrow \infty$, then $x \preceq x_{n}$ for all $n \in \mathbb{N} \cup\{0\}$;

(iv) for all $x, y \in X$ and all $t>0$ with $x \preceq y$, we have

$$
\frac{1}{M(f x, f y, t)} \leq \lambda P^{f}(x, y, t)+\left|Q^{f}(x, y, t)-\lambda\right|
$$

where $\lambda \in(0,1)$.

Then $f$ has a fixed point.

\section{Some results in non-Archimedean fuzzy metric spaces}

In this section we state and prove certain fixed point results in the setting of nonArchimedean fuzzy metric space to generalize the work of Miheț [14]. 
Theorem 3.1 Let $(X, M, \star, \preceq)$ be a partially ordered complete non-Archimedean fuzzy metric space and $f$ be an $\alpha$-admissible and non-increasing mapping. Also suppose that the following assertions hold:

(i) there exists $x_{0} \in X$ such that $\alpha\left(x_{0}, f x_{0}, t\right) \geq t$ for all $t>0$ and $x_{0} \leq f x_{0}$;

(ii) if $\left\{x_{n}\right\}$ is an increasing sequence such that $\alpha\left(x_{n}, x_{n+1}, t\right) \geq t$ for all $n \in \mathbb{N}$ and all $t>0$ with $x_{n} \rightarrow x$ as $n \rightarrow+\infty$, then $x_{n} \preceq x$ for all $n \in \mathbb{N}$ where $\alpha(x, f x, t) \geq t$;

(iii) for all comparable $x, y \in X$ and all $t>0$, we have

$$
\begin{aligned}
& {[\alpha(x, f x, t) \alpha(y, f y, t)+1]^{\psi(M(f x, f y, t))}\left[t^{2}+\frac{\alpha(x, f x, t)+\alpha(y, f y, t)}{2 t}\right]^{\phi(M(x, y, t))}} \\
& \quad \leq\left[t^{2}+1\right]^{\psi(M(x, y, t))}
\end{aligned}
$$

where $\psi, \phi:[0,1] \rightarrow[0,1]$ are two continuous functions such that $\psi$ is decreasing, $\psi(t)=0$ iff $t=1$ and $\phi(t)>0$ for all $t \in(0,1)$.

Then $f$ has a fixed point.

Proof Let $x_{0} \preceq f x_{0}$. If $x_{0}=f x_{0}$, then the result is proved. Hence we suppose that $x_{0} \prec f x_{0}$. Define a sequence $\left\{x_{n}\right\}$ by $x_{n}=f^{n} x_{0}=f x_{n-1}$ for all $n \in \mathbb{N}$. Since $f$ is non-decreasing and $x_{0} \prec f x_{0}$, then

$$
x_{0} \prec x_{1} \preceq x_{2} \preceq \cdots,
$$

and hence $\left\{x_{n}\right\}$ is a non-decreasing sequence. If $x_{n}=x_{n+1}=f x_{n}$ for some $n \in \mathbb{N}$, then the result is proved as $x_{n}$ is a fixed point of $f$. In what follows we suppose that $0<M\left(x_{n}, x_{n+1}\right.$, $t)<1$. Since $f$ is an $\alpha$-admissible mapping and

$$
\alpha\left(x_{0}, f x_{0}, t\right)=\alpha\left(x_{0}, x_{1}, t\right) \geq t,
$$

we deduce that

$$
\alpha\left(x_{1}, x_{2}, t\right)=\alpha\left(f x_{0}, f x_{1}, t\right) \geq t .
$$

Continuing this process, we get $\alpha\left(x_{n}, x_{n+1}, t\right) \geq t$ for all $n \in \mathbb{N} \cup\{0\}$ and all $t>0$. From (iii) with $x=x_{n-1}$ and $y=x_{n}$, we obtain

$$
\begin{aligned}
{\left[t^{2}+1\right]^{\psi\left(M\left(x_{n}, x_{n+1}, t\right)\right)+\phi\left(M\left(x_{n-1}, x_{n}, t\right)\right)} } & \\
= & {\left[t^{2}+1\right]^{\psi\left(M\left(x_{n}, x_{n+1}, t\right)\right)}\left[t^{2}+\frac{t+t}{2 t}\right]^{\phi\left(M\left(x_{n-1}, x_{n}, t\right)\right)} } \\
\leq & {\left[\alpha\left(x_{n-1}, x_{n}, t\right) \alpha\left(x_{n}, x_{n+1}, t\right)+1\right]^{\psi\left(M\left(x_{n-1}, f x_{n}, t\right)\right)} } \\
& \times\left[t^{2}+\frac{\alpha\left(x_{n-1}, x_{n}, t\right)+\alpha\left(x_{n}, x_{n+1}, t\right)}{2 t}\right]^{\phi\left(M\left(x_{n-1}, x_{n}, t\right)\right)} \\
\leq & {\left[t^{2}+1\right]^{\psi\left(M\left(x_{n-1}, x_{n}, t\right)\right)} }
\end{aligned}
$$

and so

$$
\psi\left(M\left(x_{n}, x_{n+1}, t\right)\right) \leq \psi\left(M\left(x_{n-1}, x_{n}, t\right)\right)-\phi\left(M\left(x_{n-1}, x_{n}, t\right)\right) \leq \psi\left(M\left(x_{n-1}, x_{n}, t\right)\right) .
$$


Since $\psi$ is decreasing, so $M\left(x_{n-1}, x_{n}, t\right) \leq M\left(x_{n}, x_{n+1}, t\right)$. Hence, $\left\{M\left(x_{n}, x_{n+1}, t\right)\right\}$ is an increasing sequence in $(0,1]$. Then there exists $l(t) \in(0,1]$ such that

$$
\lim _{n \rightarrow+\infty} M\left(x_{n}, x_{n+1}, t\right)=l(t)
$$

for all $t>0$. Let us prove that $l(t)=1$ for all $t>0$. Suppose that there exists $t_{0}>0$ such that $0<l\left(t_{0}\right)<1$. By taking the limit as $n \rightarrow+\infty$ in (3.2), we have

$$
\psi\left(l\left(t_{0}\right)\right) \leq \psi\left(l\left(t_{0}\right)\right)-\phi\left(l\left(t_{0}\right)\right) .
$$

Then $\phi\left(l\left(t_{0}\right)\right)=0$, which is a contradiction, and so $l(t)=1$ for all $t>0$. Now, we want to show that $\left\{x_{n}\right\}$ is a Cauchy sequence. Assume that it is not true. Then there exist $\epsilon \in(0,1)$ and $t_{0}>0$ such that for all $k \in \mathbb{N}$ there exist $n(k), m(k) \in \mathbb{N}$ with $m(k)>n(k) \geq k$ and

$$
M\left(x_{m(k)}, x_{n(k)}, t_{0}\right) \leq 1-\epsilon .
$$

Assume that $m(k)$ is the least integer exceeding $n(k)$ satisfying the above inequality. Equivalently,

$$
M\left(x_{m(k)-1}, x_{n(k)}, t_{0}\right)>1-\epsilon,
$$

and so for all $k$ we get

$$
\begin{aligned}
1-\epsilon & \geq M\left(x_{m(k)}, x_{n(k)}, t_{0}\right) \\
& \geq M\left(x_{m(k)-1}, x_{m(k)}, t_{0}\right) \star M\left(x_{m(k)-1}, x_{n(k)}, t_{0}\right) \\
& >\tau_{m(k)}\left(t_{0}\right) \star(1-\epsilon) .
\end{aligned}
$$

By taking limit as $n \rightarrow+\infty$ in (3.5), we deduce that

$$
\lim _{n \rightarrow+\infty} M\left(x_{m(k)}, x_{n(k)}, t_{0}\right)=1-\epsilon
$$

for $t>0$.

From

$$
\begin{aligned}
M\left(x_{m(k)+1}, x_{n(k)+1}, t_{0}\right) \geq & M\left(x_{m(k)+1}, x_{m(k)}, t_{0}\right) \star M\left(x_{m(k)}, x_{n(k)}, t_{0}\right) \\
& \star M\left(x_{n(k)}, x_{n(k)+1}, t_{0}\right)
\end{aligned}
$$

and

$$
\begin{aligned}
M\left(x_{m(k)}, x_{n(k)}, t_{0}\right) \geq & M\left(x_{m(k)+1}, x_{m(k)}, t_{0}\right) \star M\left(x_{m(k)+1}, x_{n(k)+1}, t_{0}\right) \\
& \star M\left(x_{n(k)}, x_{n(k)+1}, t_{0}\right),
\end{aligned}
$$

we get

$$
\lim _{n \rightarrow+\infty} M\left(x_{m(k)+1}, x_{n(k)+1}, t_{0}\right)=1-\epsilon .
$$


From (iii) with $x=x_{m(k)}$ and $y=x_{n(k)}$, we deduce

$$
\begin{aligned}
& {\left[t_{0}^{2}+1\right]^{\psi\left(M\left(x_{m(k)+1}, x_{n(k)+1}, t_{0}\right)\right)+\phi\left(M\left(x_{m(k)}, x_{n(k)}, t_{0}\right)\right)}} \\
& =\left[t_{0}^{2}+1\right]^{\psi\left(M\left(f x_{m(k)}, f x_{n(k)}, t_{0}\right)\right)}\left[t_{0}^{2}+\frac{t_{0}+t_{0}}{2 t_{0}}\right]^{\phi\left(M\left(x_{m(k)}, x_{n(k)}, t_{0}\right)\right)} \\
& \leq\left[\alpha\left(x_{m(k)}, f x_{m(k)}, t_{0}\right) \alpha\left(x_{n(k)}, f x_{n(k)}, t_{0}\right)+1\right]^{\psi\left(M\left(f x_{m(k)}, f x_{n(k)}, t_{0}\right)\right)} \\
& \quad \times\left[t_{0}^{2}+\frac{\alpha\left(x_{m(k)}, f x_{m(k)}, t_{0}\right)+\alpha\left(x_{n(k)}, f x_{n(k)}, t_{0}\right)}{2 t_{0}}\right]^{\phi\left(M\left(x_{m(k)}, x_{n(k)}, t_{0}\right)\right)} \\
& \leq\left[t_{0}^{2}+1\right]^{\psi\left(M\left(x_{m(k)}, x_{n(k)}, t_{0}\right)\right)},
\end{aligned}
$$

which implies

$$
\psi\left(M\left(x_{m(k)+1}, x_{n(k)+1}, t_{0}\right)\right) \leq \psi\left(M\left(x_{m(k)}, x_{n(k)}, t_{0}\right)\right)-\phi\left(M\left(x_{m(k)}, x_{n(k)}, t_{0}\right)\right) .
$$

Applying the continuity of the functions $\phi$ and $\psi$, by taking the limit as $k \rightarrow+\infty$ in the above inequality, we get

$$
\psi(1-\epsilon) \leq \psi(1-\epsilon)-\phi(1-\epsilon)
$$

and so $\phi(1-\epsilon)=0$, which is a contradiction. Then $\left\{x_{n}\right\}$ is a Cauchy sequence. Since $(X, M, \star)$ is a complete non-Archimedean fuzzy metric space, then the sequence $\left\{x_{n}\right\}$ converges to some $z \in X$, that is, for all $t>0$,

$$
\lim _{n \rightarrow+\infty} M\left(x_{n}, z, t\right)=1
$$

Assume that there exists $t_{0}>0$ such that $0<M\left(z, f z, t_{0}\right)<1$. Then by (2.11) and (ii) we get

$$
\begin{aligned}
& {\left[t_{0}^{2}+1\right]^{\psi\left(M\left(x_{n+1}, f z, t_{0}\right)\right)+\phi\left(M\left(x_{n}, z, t_{0}\right)\right)}} \\
& \quad=\left[t_{0}^{2}+1\right]^{\psi\left(M\left(f x_{n}, f z, t_{0}\right)\right)}\left[t_{0}^{2}+\frac{t_{0}+t_{0}}{2 t_{0}}\right]^{\phi\left(M\left(x_{n}, z, t_{0}\right)\right)} \\
& \quad \leq\left[\alpha\left(x_{n}, f x_{n}, t_{0}\right) \alpha\left(z, f z, t_{0}\right)+1\right]^{\psi\left(M\left(f x_{n}, f z, t_{0}\right)\right)}\left[t_{0}^{2}+\frac{\alpha\left(x_{n}, f x_{n}, t_{0}\right)+\alpha\left(z, f z, t_{0}\right)}{2 t_{0}}\right]^{\phi\left(M\left(x_{n}, z, t_{0}\right)\right)} \\
& \quad \leq\left[t_{0}^{2}+1\right]^{\psi\left(M\left(x_{n}, z, t_{0}\right)\right)}
\end{aligned}
$$

and hence

$$
\psi\left(M\left(x_{n+1}, f z, t_{0}\right)\right) \leq \psi\left(M\left(x_{n}, z, t_{0}\right)\right)-\phi\left(M\left(x_{n}, z, t_{0}\right)\right)
$$

By taking the limit as $n \rightarrow+\infty$ in the above inequality, we have

$$
\psi\left(M\left(z, f z, t_{0}\right)\right) \leq \psi(1)-\phi(1) \leq \psi(1)=0
$$

Then $\psi\left(M\left(z, f z, t_{0}\right)\right)=0$, i.e., $M\left(z, f z, t_{0}\right)=1$, which is a contradiction. Hence, $M(z, f z, t)=1$ for all $t>0$, that is, $z=f z$. 
If in Theorem 3.1 we take $\alpha(x, y, t)=t$ for all $x, y \in X$ and all $t>0$, then we deduce the following corollary.

Corollary 3.1 Let $(X, M, \star, \preceq)$ be a partially ordered complete non-Archimedean fuzzy metric space, $\psi, \phi:[0,1] \rightarrow[0,1]$ as in Theorem 3.1 and $f: X \rightarrow X$ be an increasing mapping such that

$$
\psi(M(f x, f y, t)) \leq \psi(M(x, y, t))-\phi(M(x, y, t))
$$

holds for all comparable $x, y \in X$. If the following assertions hold:

(i) there exists $x_{0} \in X$ such that $x_{0} \preceq f x_{0}$;

(ii) if $\left\{x_{n}\right\}$ is an increasing sequence such that $x_{n} \rightarrow x$ as $n \rightarrow+\infty$, then $x_{n} \preceq x$ for all $n \in \mathbb{N}$.

Then $f$ has a fixed point.

Theorem 3.2 Let $(X, M, \star, \preceq)$ be a partially ordered complete non-Archimedean fuzzy metric space and $f$ be an $\alpha$-admissible and non-increasing mapping such that the following assertions hold:

(i) there exists $x_{0} \in X$ such that $\alpha\left(x_{0}, f x_{0}, t\right) \geq t$ for all $t>0$ and $x_{0} \leq f x_{0}$;

(ii) if $\left\{x_{n}\right\}$ is an increasing sequence such that $\alpha\left(x_{n}, x_{n+1}, t\right) \geq t$ for all $n \in \mathbb{N} \cup\{0\}$, all $t>0$ and $x_{n} \rightarrow x$ as $n \rightarrow+\infty$, then $x_{n} \preceq x$ for all $n \in \mathbb{N}$ where $\alpha(x, f x, t) \geq t$;

(iii) assume that there exists a function $\beta:[0,1] \rightarrow[1,+\infty)$ such that for any sequence $\left\{t_{n}\right\} \subseteq[0,1]$ of positive reals, $\beta\left(t_{n}\right) \rightarrow 1$ implies $t_{n} \rightarrow 1$ such that

$$
\begin{aligned}
& {\left[t^{2}+1\right]^{M(f x, f y, t)}} \\
& \quad \geq\left[\frac{\alpha(x, f x, t) \alpha(y, f y, t)}{t^{2}}\right][\alpha(x, f x, t) \alpha(y, f y, t)+1]^{\beta(M(x, y, t)) M(x, y, t)}
\end{aligned}
$$

holds for all comparable $x, y \in X$ and all $t>0$.

Then $f$ has a fixed point.

Proof Let $x_{0} \preceq f x_{0}$. If $x_{0}=f x_{0}$, then the result is proved. Hence we suppose that $x_{0} \prec f x_{0}$. Define a sequence $\left\{x_{n}\right\}$ by $x_{n}=f^{n} x_{0}=f x_{n-1}$ for all $n \in \mathbb{N}$. Since $f$ is non-decreasing and $x_{0} \prec f x_{0}$, then

$$
x_{0} \prec x_{1} \preceq x_{2} \preceq \cdots,
$$

and hence $\left\{x_{n}\right\}$ is a non-decreasing sequence. If $x_{n}=x_{n+1}=f x_{n}$ for some $n \in \mathbb{N}$, then the result is proved as $x_{n}$ is a fixed point of $f$. In what follows we suppose that $0<M\left(x_{n}, x_{n+1}\right.$, $t)<1$. Since $f$ is an $\alpha$-admissible mapping with respect to $\eta$ and $\alpha\left(x_{0}, f x_{0}, t\right)=\alpha\left(x_{0}, x_{1}, t\right) \geq$ $t$, we deduce that $\alpha\left(x_{1}, x_{2}, t\right)=\alpha\left(f x_{0}, f x_{1}, t\right) \geq t$. By continuing this process, we get $\alpha\left(x_{n}\right.$, $\left.x_{n+1}, t\right) \geq t$ for all $n \in \mathbb{N} \cup\{0\}$ and all $t>0$. From (3.6) we get

$$
\begin{aligned}
& {\left[t^{2}+1\right]^{M\left(f x_{n-1} f x_{n}, t\right)}} \\
& \quad \geq\left[\frac{\alpha\left(x_{n-1}, f x_{n-1}, t\right) \alpha\left(x_{n}, f x_{n}, t\right)}{t^{2}}\right]
\end{aligned}
$$




$$
\begin{aligned}
& \times\left[\alpha\left(x_{n-1}, f x_{n-1}, t\right) \alpha\left(x_{n}, f x_{n}, t\right)+1\right]^{\beta\left(M\left(x_{n-1}, x_{n}, t\right)\right) M\left(x_{n-1}, x_{n}, t\right)} \\
\geq & {\left[\frac{t \cdot t}{t^{2}}\right][t \cdot t+1]^{\beta\left(M\left(x_{n-1}, x_{n}, t\right)\right) M\left(x_{n-1}, x_{n}, t\right)} } \\
= & {\left[t^{2}+1\right]^{\beta\left(M\left(x_{n-1}, x_{n}, t\right)\right) M\left(x_{n-1}, x_{n}, t\right)} . }
\end{aligned}
$$

Thus

$$
M\left(f x_{n-1}, f x_{n}, t\right) \geq \beta\left(M\left(x_{n-1}, x_{n}, t\right)\right) M\left(x_{n-1}, x_{n}, t\right) .
$$

Hence,

$$
M\left(x_{n}, x_{n+1}, t\right) \geq \beta\left(M\left(x_{n-1}, x_{n}, t\right)\right) M\left(x_{n-1}, x_{n}, t\right) \geq M\left(x_{n-1}, x_{n}, t\right) .
$$

That is, $\left\{S_{n}=M\left(x_{n}, x_{n+1}, t\right)\right\}$ is an increasing sequence in $(0,1]$. Then there exists $l(t) \in(0,1]$ such that $\lim _{n \rightarrow+\infty} M\left(x_{n}, x_{n+1}, t\right)=l(t)$ for all $t>0$. We shall prove that $l(t)=1$ for all $t>0$. By (3.8) we deduce

$$
\frac{M\left(x_{n}, x_{n+1}, t\right)}{M\left(x_{n-1}, x_{n}, t\right)} \geq \beta\left(M\left(x_{n-1}, x_{n}, t\right)\right) \geq 1,
$$

which implies $\lim _{n \rightarrow+\infty} \beta\left(M\left(x_{n-1}, x_{n}, t\right)\right)=1$. Regarding the property of the function $\beta$, we conclude that

$$
\lim _{n \rightarrow+\infty} M\left(x_{n}, x_{n+1}, t\right)=1
$$

Next, we shall prove that $\left\{x_{n}\right\}$ is a Cauchy sequence. Suppose, to the contrary, that $\left\{x_{n}\right\}$ is not a Cauchy sequence. Proceeding as in the proof of Theorem 3.1, there exist $\epsilon \in(0,1)$ and $t_{0}>0$ such that for all $k \in \mathbb{N}$ there exist $n(k), m(k) \in \mathbb{N}$ with $m(k)>n(k) \geq k$ such that

$$
\lim _{n \rightarrow+\infty} M\left(x_{m(k)}, x_{n(k)}, t_{0}\right)=1-\epsilon
$$

and

$$
\lim _{n \rightarrow+\infty} M\left(x_{m(k)+1}, x_{n(k)+1}, t_{0}\right)=1-\epsilon .
$$

From (3.6) with $x=x_{m(k)}$ and $y=x_{n(k)}$ we deduce

$$
\begin{aligned}
{\left[t^{2}+1\right]^{M\left(f x_{m(k)}, f x_{n(k)}, t_{0}\right)} } & \\
\geq & {\left[\frac{\alpha\left(x_{m(k)}, f x_{m(k)}, t\right) \alpha\left(x_{n(k)}, f x_{n(k)}, t\right)}{t^{2}}\right] } \\
& \times\left[\alpha\left(x_{m(k)}, f x_{m(k)}, t\right) \alpha\left(x_{n(k)}, f x_{n(k)}, t\right)+1\right]^{\beta\left(M\left(x_{m(k)}, x_{n(k)}, t\right)\right) M\left(x_{m(k)}, x_{n(k)}, t_{0}\right)} \\
\geq & {\left[\frac{t \cdot t}{t^{2}}\right]\left[t^{2}+1\right]^{\beta\left(M\left(x_{m(k)}, x_{n(k)}, t\right)\right) M\left(x_{m(k)}, x_{n(k)}, t_{0}\right)} } \\
= & {\left[t^{2}+1\right]^{\beta\left(M\left(x_{m(k)}, x_{n(k)}, t\right)\right) M\left(x_{m(k)}, x_{n(k)}, t_{0}\right)}, }
\end{aligned}
$$


which implies

$$
M\left(f x_{m(k)}, f x_{n(k)}, t_{0}\right) \geq \beta\left(M\left(x_{m(k)}, x_{n(k)}, t\right)\right) M\left(x_{m(k)}, x_{n(k)}, t_{0}\right),
$$

and so

$$
\frac{M\left(x_{m(k)+1}, x_{n(k)+1}, t_{0}\right)}{M\left(x_{m(k)}, x_{m(k)}, t_{0}\right)} \geq \beta\left(M\left(x_{m(k)}, x_{m(k)}, t_{0}\right)\right) \geq 1 .
$$

Taking the limit as $k \rightarrow+\infty$ in the above inequality, we get

$$
\lim _{k \rightarrow+\infty} \beta\left(M\left(x_{m(k)}, x_{n(k)}, t_{0}\right)\right)=1
$$

which implies

$$
1-\epsilon=\lim _{k \rightarrow+\infty} M\left(x_{m(k)}, x_{n(k)}, t_{0}\right)=1,
$$

and so $\epsilon=0$, which is a contradiction. Then $\left\{x_{n}\right\}$ is a Cauchy sequence. Since $(X, M, \star)$ is a complete space, then the sequence $\left\{x_{n}\right\}$ converges to some $z \in X$ such that for all $t>0$,

$$
\lim _{n \rightarrow+\infty} M\left(x_{n}, z, t\right)=1
$$

By (3.6) and (ii) we get

$$
\begin{aligned}
{\left[t^{2}+1\right]^{M\left(f x_{n}, f z, t\right)} } & \geq\left[\frac{\alpha\left(x_{n}, f x_{n}, t\right) \alpha(z, f z, t)}{t^{2}}\right]\left[\alpha\left(x_{n}, f x_{n}, t\right) \alpha(z, f z, t)+1\right]^{\beta\left(M\left(x_{n}, z, t\right)\right) M\left(x_{n}, z, t\right)} \\
& \geq\left[\frac{t \cdot t}{t^{2}}\right]\left[t^{2}+1\right]^{M\left(x_{n}, z, t\right)} \\
& =\left[t^{2}+1\right]^{M\left(x_{n}, z, t\right)}
\end{aligned}
$$

and hence

$$
M\left(x_{n}, f z, t\right) \geq M\left(x_{n}, z, t\right) .
$$

Taking the limit as $n \rightarrow+\infty$ in the above inequality, we have $\lim _{n \rightarrow+\infty} M\left(f x_{n}, f z, t\right)=1$ for all $t>0$ and then

$$
M(z, f z, t) \geq \lim _{n \rightarrow+\infty} M\left(f x_{n}, z, t\right) \star \lim _{n \rightarrow \infty} M\left(f x_{n}, f z, t\right)=1 \star 1=1,
$$

that is, $z=f z$.

Example 3.1 Let $(X, M, \star)$ be the non-Archimedean fuzzy metric space where $M(x, y, t)=$ $\frac{\min \{x, y\}}{\max \{x, y\}}$ for all $t>0$ and $a \star b=\min \{a, b\}$. Define $f: X \rightarrow X$ with

$$
f x= \begin{cases}\frac{1}{3} x & \text { if } x \in[1,3], \\ \ln (x-3)+1 & \text { if } x \in(3,+\infty) .\end{cases}
$$

Also define $\alpha(x, y, t)=\left\{\begin{array}{ll}t, & \text { if } x, y \in[1,3], \\ 0, & \text { otherwise }\end{array}\right.$ and $\beta(t)=1$. Also, $x \preceq y$ iff $x \leq y$. 
Let, $x, y \in[1,3]$ and $x \leq y$. Then

$$
\begin{aligned}
{\left[t^{2}+1\right]^{M(f x, f y, t)} } & =2^{\left(\frac{x}{y}\right)} \geq 2^{\left(\frac{x}{y}\right)} \\
& =\left[\frac{\alpha(x, f x, t) \alpha(y, f y, t)}{2 t}\right][\alpha(x, f x, t) \alpha(y, f y, t)+1]^{\beta(M(x, y, t)) M(x, y, t)} .
\end{aligned}
$$

Otherwise, $\alpha(x, f x, t) \alpha(y, f y, t)=0$, and so

$$
\left[t^{2}+1\right]^{M(f x, f y, t)} \geq 0=\left[\frac{\alpha(x, f x, t) \alpha(y, f y, t)}{2 t}\right][\alpha(x, f x, t) \alpha(y, f y, t)+1]^{\beta(M(x, y, t)) M(x, y, t)} .
$$

Clearly, $\alpha(1, f 1, t) \geq t$ and $1 \leq f 1$. Now, if $\left\{x_{n}\right\}$ is an increasing sequence in $X$ such that $\alpha\left(x_{n}, x_{n+1}, t\right) \geq 1$ for all $n \in \mathbb{N} \cup\{0\}$ and $x_{n} \rightarrow x$ as $n \rightarrow \infty$, then $\left\{x_{n}\right\} \subset[0,1]$ and hence $x \in[0,1]$. This implies that $x_{n} \rightarrow x$ for all $n \in \mathbb{N}$ and $\alpha(x, f x, t) \geq t$. Hence, all the conditions of Theorem 3.2 hold and $f$ has a fixed point. Then, by Theorem 3.2, $f$ has a fixed point.

If in Theorem 3.2 we take $\alpha(x, y, t)=t$ for all $x, y \in X$, then we deduce the following result.

Corollary 3.2 Let $(X, M, \star, \preceq)$ be a complete non-Archimedean fuzzy metric space, and $f$ be an increasing mapping on $X$. Assume that there exists a function $\beta:[0,1] \rightarrow[1,+\infty)$ such that for any sequence $\left\{t_{n}\right\} \subseteq[0,1]$ of positive reals, $\beta\left(t_{n}\right) \rightarrow 1$ implies $t_{n} \rightarrow 1$ and

$$
M(f x, f y, t) \geq \beta(M(x, y, t)) M(x, y, t)
$$

for all comparable $x, y \in X$ and all $t>0$. Also suppose that the following assertions hold:

(i) there exists $x_{0} \in X$ such that $x_{0} \preceq f x_{0}$;

(ii) if $\left\{x_{n}\right\}$ is an increasing sequence such that $x_{n} \rightarrow x$ as $n \rightarrow+\infty$, then $x_{n} \preceq x$ for all $n \in \mathbb{N}$.

Then $f$ has a fixed point.

\section{Application to integral equations}

Fixed point theorems for monotone operators in ordered metric spaces are widely investigated and have found various applications in differential and integral equations (see [27$31]$ and the references therein). Let $X=C([0, T], \mathbb{R})$ be the set of real continuous functions defined on $[0, T]$ and $M: X \times X \times(0,+\infty) \rightarrow[0,1]$ be defined by

$$
M(x, y, r)=\frac{r}{r+\|x-y\|_{\infty}}
$$

for all $x, y \in X$ and all $r>0$. Also define $a \star b=\min \{a, b\}$. Then $(M, X, \star)$ is a complete triangular fuzzy metric space.

Consider the integral equation

$$
x(t)=p(t)+\int_{0}^{T} S(t, s) f(s, x(s)) d s
$$

and the mapping $F: X \rightarrow X$ defined by

$$
F x(t)=p(t)+\int_{0}^{T} S(t, s) f(s, x(s)) d s,
$$


where

(A) $f:[0, T] \times \mathbb{R} \rightarrow \mathbb{R}$ is continuous;

(B) $p:[0, T] \rightarrow \mathbb{R}$ is continuous;

(C) $S:[0, T] \times[0, T] \rightarrow[0,+\infty)$ is continuous;

(D) there exist $\theta: X \times X \rightarrow \mathbb{R}$ and $\lambda \in(0,1)$ such that if $\theta(x, y) \geq 0$ for $x, y \in X$, then for all $s \in[0, T]$ and all $r>0$ we have

$$
\begin{aligned}
|f(s, x(s))-f(s, y(s))| \leq & \lambda \max \{|x-y|,|x(s)-F x(s)|,|y(s)-F y(s)|\} \\
& +r\left|Q^{F}(x, y, r)-\lambda\right|+\lambda r-r ;
\end{aligned}
$$

$(\mathrm{F})$

$$
\int_{0}^{T} S(t, s) d s \leq 1 \quad \text { for all } t \in[0, T]
$$

(G) there exists $x_{0} \in X$ such that $\theta\left(x_{0}, F x_{0}\right) \geq 0$;

$(\mathrm{H})$

$$
\theta(x, y) \geq 0 \quad \text { for some } x \in X \quad \text { implies } \quad \theta(F x, F y) \geq 0
$$

(I) if $\left\{x_{n}\right\}$ is a sequence in $X$ such that $\theta\left(x_{n}, x_{n+1}\right) \geq 0$ for all $n \in \mathbb{N} \cup\{0\}$ and $x_{n} \rightarrow x$ as $n \rightarrow+\infty$, then $\theta\left(x_{n}, x\right) \geq 0$.

Theorem 4.1 Under the assumptions (A)-(I), the integral equation (4.1) has a solution in $X=C([0, T], \mathbb{R})$.

Proof Let $F: X \rightarrow X$ be defined by (4.2) and let $x, y \in X$ be such that $\theta(x, y) \geq 0$. By the condition (D), we deduce that

$$
\begin{aligned}
|F x(t)-F y(t)|= & \left|\int_{0}^{T} S(t, s)[f(s, x(s))-f(s, y(s))] d s\right| \\
\leq & \int_{0}^{T} S(t, s)|f(s, x(s))-f(s, y(s))| d s \\
\leq & \int_{0}^{T} S(t, s)[\lambda \max \{|x-y|,|x(s)-F x(s)|,|y(s)-F y(s)|\} \\
& \left.+r\left|Q^{F}(x, y, r)-\lambda\right|+\lambda r-r\right] d s \\
\leq & \int_{0}^{T} S(t, s)\left[\lambda \max \left\{\|x-y\|_{\infty},\|x(s)-F x(s)\|_{\infty},\|y(s)-F y(s)\|_{\infty}\right\}\right. \\
& \left.+r\left|Q^{F}(x, y, r)-\lambda\right|+\lambda r-r\right] d s \\
= & {\left[\lambda \max \left\{\|x-y\|_{\infty},\|x(s)-F x(s)\|_{\infty},\|y(s)-F y(s)\|_{\infty}\right\}\right.} \\
& \left.+r\left|Q^{F}(x, y, r)-\lambda\right|+\lambda r-r\right]\left(\int_{0}^{T} S(t, s) d s\right) \\
\leq & \lambda \max \left\{\|x-y\|_{\infty},\|x(s)-F x(s)\|_{\infty},\|y(s)-F y(s)\|_{\infty}\right\} \\
& +r\left|Q^{F}(x, y, r)-\lambda\right|+\lambda r-r
\end{aligned}
$$




$$
\begin{aligned}
= & \lambda r \max \left\{\frac{1}{r}\|x-y\|_{\infty}, \frac{1}{r}\|x(s)-F x(s)\|_{\infty}, \frac{1}{r}\|y(s)-F y(s)\|_{\infty}\right\} \\
& +r\left|Q^{F}(x, y, r)-\lambda\right|+\lambda r-r \\
= & \lambda r \max \left\{\frac{1}{r}\|x-y\|_{\infty}+1, \frac{1}{r}\|x-F x\|_{\infty}+1, \frac{1}{r}\|y-F y\|_{\infty}+1\right\} \\
& +r\left|Q^{F}(x, y, r)-\lambda\right|+\lambda r-r-\lambda r \\
= & \lambda r \max \left\{\frac{1}{M(x, y, r)}, \frac{1}{M(x, F x, r)}, \frac{1}{M(y, F y, r)}\right\} \\
& +r\left|Q^{F}(x, y, r)-\lambda\right|-r \\
\leq & \lambda r P^{F}(x, y, r)+r\left|Q^{F}(x, y, r)-\lambda\right|-r,
\end{aligned}
$$

and so

$$
\|F x-F y\|_{\infty} \leq \lambda r P^{f}(x, y, r)+r\left|Q^{f}(x, y, r)-\lambda\right|-r .
$$

Now we define $\alpha: X \times(0, \infty) \rightarrow[0,+\infty)$ by

$$
\alpha(x, y, r)= \begin{cases}r, & \text { if } \theta(x, y) \geq 0 \\ 0, & \text { otherwise }\end{cases}
$$

Since $\theta(x, y) \geq 0$, so, $\alpha(x, y, r)=r$. Therefore we can write

$$
\begin{aligned}
\frac{\alpha(x, y, r)}{r M(F x, F y, r)} & =\frac{r}{r M(F x, F y, r)}=\frac{1}{M(F x, F y, r)}=\frac{1}{r}\|F x-F y\|_{\infty}+1 \\
& \leq \frac{1}{r}\left(\lambda r P^{F}(x, y, r)+r\left|Q^{F}(x, y, r)-\lambda\right|-r\right)+1 \\
& =\lambda P^{F}(x, y, r)+\left|Q^{F}(x, y, r)-\lambda\right|-1+1 \\
& =\lambda P^{F}(x, y, r)+\left|Q^{F}(x, y, r)-\lambda\right| .
\end{aligned}
$$

Thus all of the conditions of Corollary 2.2 are satisfied and hence the mapping $F$ has a fixed point which is a solution of the integral equation $(4.1)$ in $X=C([0, T], \mathbb{R})$.

\section{Competing interests}

The authors declare that they have no competing interests.

\section{Authors' contributions}

All authors contributed equally and significantly in writing this article. All authors read and approved the final manuscript.

\section{Author details}

${ }^{1}$ Department of Mathematics, King Abdulaziz University, P.O. Box 80203, Jeddah, 21589, Saudi Arabia. ${ }^{2}$ Department of Mathematics, Payame Noor University (PNU), P.O. Box 19395-3697, Tehran, Iran. ${ }^{3}$ Young Researchers and Elite Club, Rasht Branch, Islamic Azad University, Rasht, Iran.

\section{Acknowledgements}

This article was funded by the Deanship of Scientific Research (DSR), King Abdulaziz University, Jeddah. Therefore, the first author acknowledges with thanks DSR, KAU for financial support.

Received: 20 April 2015 Accepted: 18 July 2015 Published online: 01 August 2015 


\section{References}

1. Deng, Z-K: Fuzzy pseudometric spaces. J. Math. Anal. Appl. 86, 74-95 (1982)

2. Kramosil, I, Michálek, J: Fuzzy metrics and statistical metric spaces. Kybernetika 11, 336-344 (1975)

3. Di Bari, C, Vetro, C: A fixed point theorem for a family of mappings in a fuzzy metric space. Rend. Circ. Mat. Palermo 52, 315-321 (2003)

4. Di Bari, C, Vetro, C: Fixed points, attractors and weak fuzzy contractive mappings in a fuzzy metric space. J. Fuzzy Math. 13, 973-982 (2005)

5. Gopal, D, Imdad, M, Vetro, C, Hasan, M: Fixed point theory for cyclic weak $\phi$-contraction in fuzzy metric spaces. J. Nonlinear Anal. Appl. 2012, Article ID jnaa-00110 (2012)

6. Grabiec, M: Fixed points in fuzzy metric spaces. Fuzzy Sets Syst. 27, 385-389 (1988)

7. Gregori, V, Sapena, A: On fixed-point theorems in fuzzy metric spaces. Fuzzy Sets Syst. 125, 245-252 (2002)

8. Hadžić, O, Pap, E: Fixed Point Theory in Probabilistic Metric Spaces. Kluwer Academic, London (2001)

9. Miheț, D: A class of contractions in fuzzy metric spaces. Fuzzy Sets Syst. 161, 1131-1137 (2010)

10. Vetro, C, Gopal, D, Imdad, M: Common fixed point theorem for $(\phi, \psi)$-weak contractions in fuzzy metric spaces. Indian J. Math. 52, 573-590 (2010)

11. Vetro, C, Vetro, P: Common fixed points for discontinuous mappings in fuzzy metric spaces. Rend. Circ. Mat. Palermo 57, 295-303 (2008)

12. George, A, Veeramani, P: On some results in fuzzy metric spaces. Fuzzy Sets Syst. 64, 395-399 (1994)

13. George, A, Veeramani, P: On some results of analysis for fuzzy metric spaces. Fuzzy Sets Syst. 90, 365-368 (1997)

14. Miheț, D: Fuzzy $\psi$-contractive mappings in non-Archimedean fuzzy metric spaces. Fuzzy Sets Syst. 159, 739-744 (2008)

15. Vetro, C: Fixed points in weak non-Archimedean fuzzy metric spaces. Fuzzy Sets Syst. 162, 84-90 (2011)

16. Pathak, HK, Hussain, N: Common fixed points for Banach pairs with applications. Nonlinear Anal. 69, 2788-2802 (2008)

17. Samet, B, Vetro, C, Vetro, P: Fixed point theorems for $\alpha$ - $\psi$-contractive type mappings. Nonlinear Anal. 75, 2154-2165 (2012)

18. Salimi, P, Latif, A, Hussain, N: Modified $\alpha$ - $\psi$-contractive mappings with applications. Fixed Point Theory Appl. 2013 , 151 (2013)

19. Hussain, N, Ahmad, J, Azam, A: On Suzuki-Wardowski type fixed point theorems. J. Nonlinear Sci. Appl. 8, 1095-1111 (2015)

20. Hussain, $N$, Salimi, $P$, Latif, A: Fixed point results for single and set-valued $\alpha-\eta-\psi$-contractive mappings. Fixed Point Theory Appl. 2013, 212 (2013)

21. Hussain, N, Kutbi, MA, Khaleghizadeh, S, Salimi, P: Discussions on recent results for $\alpha$ - $\psi$-contractive mappings. Abstr. Appl. Anal. 2014, Article ID 456482 (2014)

22. Hussain, N, Khaleghizadeh, S, Salimi, P, Abdou, AAN: A new approach to fixed point results in triangular intuitionistic fuzzy metric spaces. Abstr. Appl. Anal. 2014, Article ID 690139 (2014)

23. Hussain, N, Khaleghizadeh, S, Salimi, P, Akbar, F: New fixed point results with PPF dependence in Banach spaces endowed with a graph. Abstr. Appl. Anal. 2013, Article ID 827205 (2013)

24. Karapinar, E, Samet, B: Generalized $\alpha$ - $\psi$-contractive type mappings and related fixed point theorems with applications. Abstr. Appl. Anal. 2012, Article ID 793486 (2012)

25. Parvaneh, V, Salimi, P, Vetro, P, Nezhad, AD, Radenović, S: Fixed point results for GP contractive mappings. J. Nonlinear Sci. Appl. 7, 150-159 (2014)

26. Latif, A, Hezarjaribi, M, Salimi, P, Hussain, N: Best proximity point theorems for $\alpha$ - $\psi$-proximal contractions in intuitionistic fuzzy metric spaces. J. Inequal. Appl. 2014, 352 (2014)

27. Agarwal, RP, Hussain, N, Taoudi, MA: Fixed point theorems in ordered Banach spaces and applications to nonlinear integral equations. Abstr. Appl. Anal. 2012, Article ID 245872 (2012)

28. Hussain, N, Khan, AR, Agarwal, RP: Krasnosel'skii and Ky Fan type fixed point theorems in ordered Banach spaces. J. Nonlinear Convex Anal. 11(3), 475-489 (2010)

29. Hussain, N, Taoudi, MA: Krasnosel'skii-type fixed point theorems with applications to Volterra integral equations. Fixed Point Theory Appl. 2013, 196 (2013)

30. De la Sen, M, Karapinar, E: On a cyclic Jungck modified TS-iterative procedure with application examples. Appl. Math. Comput. 233, 383-397 (2014)

31. Nieto, JJ, Rodríguez-López, R: Contractive mapping theorems in partially ordered sets and applications to ordinary differential equations. Order 22, 223-239 (2005)

\section{Submit your manuscript to a SpringerOpen ${ }^{\odot}$ journal and benefit from:}

- Convenient online submission

- Rigorous peer review

- Immediate publication on acceptance

- Open access: articles freely available online

- High visibility within the field

- Retaining the copyright to your article 